who are more conversant with the windowed style user interface of modem personal computers and workstations. NRCVAX has therefore been redesigned to take advantage of cument graphical user interfaces (GUIs). In doing so we have been forced to make a choice of operating systens to support, as GUls tend to be linked quite intimately to the underlying operating system. We have initially chosen the Windows 95 and NT systems to be followed closely by a Motif version for Unix workstations.

A common method of providing a GUI, which works well for much code, is to write a program that interacts with the user and generates a file of input instructions for the existing routine. The routine is then executed in the background and its output trapped and presented to the user. This works well for 'batch' style software but was not suited to NRCVAX where the questions a user is asked are often based on prior responses. So the programs have been designed with the user interaction localized to a limited number of subroutines which are called directly by the user interface. Output in most programs is returned to a scrollable window which the user may choose to save or print.

As part of this effort the existing command line version of the software has been carefully examined and modified to reduce user input and eliminate redundant questions.

\section{Computing III}

\section{Macromolecular Map Fitting And Modification}

MS03.03.01 DENSITY MODTFICATION: SUCCESSESAND LIMITATIONS. Kevin Cowtan, Protein Structure Group. Dept. of Chemistry, University of York, Helsington, York Y01 5DD, England

Phase improvement by density modification, despite a number of theoretical flaws, is now an established part of the solution of macro-molecular structures by $x$-ray diffraction methods. The power of the method in the best cases has been demonstrated repeatedly by the solution of structures from the weakest of phase information, for example from single derivatives or weak anomalous scattering. If there is sufficient redundancy in the data, due to high solvent content, local symmetry or multiple crystal forms, then the method can come close to ab-initio phasing.

This success arises despite a number of fundamental flaws in the underlying protocol of most density modification calculations. In particular, the density modification 'model' is usually underdetermined with respect to the observed data. This leads to degrees of freedom in the model magnitudes which in turn invalidate the assumptions behind the usual phase combination methods.

Future developments must address these problems. Particular areas under study are the integration of model building and density modification into a semi-automatic process. At a more fundamental level, statistical phasing (maximum entropy) methods may lead to a new formulation with a firmer mathematical foundation.

MS03.03.02 ELECTRON DENSITY AVERAGING USING MULTIPLE CRYSTAL FORMS OR DIFFRACTION DATASETS IN STRUCTURE DETERMINATION OF PROTEIN AT MODERATE RESOLUTION. Kalyan Das, Jianping Ding, Yu Hsiou, Karen Lentz, Wanyi Zhang, and Edward Arnold Center for Advanced Biotechnology and Medicine (CABM) and Rutgers University Chemistry Department, 679 Hoes Lane, Piscataway, NJ 08854

Obtaining accurate phases and hence reliable structures using moderate resolution data remains challenging. In the presence of non-crystallographic symmetry (NCS), electron density map averaging techniques have been successfully used to improve the quality of phases. However, many proteins form crystals without useful noncrystallographic redundancy in the asymmetric unit. A multiple electron density maps averaging technique has been developed to improve the phase quality and the interpretability of electron density maps. We averaged the electron density maps computed from structures of HIV-1 reverse transcriptase (RT) crystallized in different crystal forms (i.e., with dramatically different unit cells or space groups). We also averaged the electron density maps calculated from multiple diffraction datasets collected from the same crystal form at different temperatures. For example, the unit cell parameters were slightly different for the crystal frozen at $-165^{\circ} \mathrm{C}$ and the same crystal cooled at $-10^{\circ} \mathrm{C}$. In this averaging technique, the protein structures are subdivided into segments that can be superimposed well and the averaging is carried out over the masks covering the segments. The averaged electron density maps for individual subdomains are then combined to cover the whole protein. Also, to minimize the model bias, the conventional omit maps were calculated and used as the input maps for averaging.

This technique was applied to the structure determination of HIV-1 RT in complexes with various nonnucleoside inhibitors (NNRTIs) at $3.0 \AA$ resolution, unliganded HIK1 RT in two crystal forms at $2.7 \AA$ and $3.5 \AA$ resolution, respectively and in the structure refinement of the HIV-1 RT/DNA/Fab complex at $2.8 \AA$ resolution. The omit maps and the averaging of the multiple electron density maps have significantly reduced the model bias and improved the interpretability of electron density. The improvement of the phases after map averaging are evident from comparison of electron densities for the bound inhibitors calculated at different stages of HIV-1 RT/NNRTI structure refinements. A detailed description of the algorithm and the results will be discussed. This approach will be useful in solving various other protein structures without NCS symmetry,

MS03.03.03 PARTIAL STRUCTURE REFINEMENT COMBINED WITH ELECTRON DENSITY MODIFICATIONS BY USING MAIN. Dusan Turk, dusan.turk@ijs.si, University of Ljubljana, Josef Stefan Institute, Slovenia

In the cases, that is, in most cases, when it is not possible to build almost complete macromolecular models into initial electron density maps, progress of a structure determination is hindered by limitations of a partial model refinement against a full data set and by limitations of electron density modification procedures including phase combination. A phase combination procedure starts to make sense when substantial parts of the model have been built into electron density maps.

MAIN is a program that can be applied in various electron density modification approaches, for interactive model building and structure refinement. Now, tools that allow to combine all these methods into a single task, have been added to the program. The essence of the idea is that accordingly scaled electron density map of a partial model is scaled and merged with a background map. Resulting merged map can be subjected to any electron density modification procedure. This modified map serves then to calculate structure factors and these structure factors are used to calculated derivatives, which are applied during a partial model minimization, and to generate a new background map. This should be repeated in several cycles.

The approach is not limited by the size of a model fraction, although it is evident that small model fractions will not be capable of substantial map improvements. Visual inspection has revealed that the resulting maps are sharper, include more structural details (side chains and loops) and are less model biased than a map based on a phase combination approach.

A MAIN demonstration installation is available via an anonymous FTP server at STEF.IJS.SI. 